\title{
Diagnosticando e tratando depressão no paciente com doença clínica
}

\author{
Diagnosing and treating depression in the medically ill
}

\author{
Letícia Maria Furlanetto e Marco Antonio Brasil
}

\begin{abstract}
Resumo
Diagnosticar depressão em um doente clínico é uma tarefa difícil por vários motivos. Muito embora tristeza e humor depressivo possam indicar síndrome depressiva subjacente, eles também podem fazer parte da adaptação normal a uma doença que ameace a vida. Os sintomas somáticos e vegetativos incluídos nos critérios diagnósticos das classificações atuais não são específicos e podem de fato ser atribuíveis à hospitalização, aos tratamentos ou à própria doença clínica. Tratar a depressão no paciente clínico é também difícil devido à preocupação com a interação de drogas, assim como questões de eficácia e segurança nesse grupo. Nesta conferência clínica os autores discutem questões como que diagnósticos podem ser feitos em pacientes clínicos com sintomas depressivos; prevalência de depressão em diferentes ambientes; mecanismos de co-morbidade (fatores fisiológicos e comportamentais) que relacionam a depressão às doenças clínicas e 0 manuseio da depressão nesse grupo.
\end{abstract}

\begin{abstract}
Diagnosing depression in the medically ill is a difficult diagnostic task for several reasons. Although sadness and depressed mood may be indicative of an underlying depressive syndrome, they may also be part of normal adaptation to a life-threatening disease. The somatic and vegetative symptoms included in diagnostic criteria are not specific and may in fact be attributable to hospitalization, treatments or medical illness. Treating depression is also difficult, because of concerns on drug-drug interactions and efficacy and safety of antidepressants in this group. In this Clinical Conference the authors answer questions on: which diagnoses can be made in medical patients with depressive symptoms; prevalence of depression in different settings; comorbidity mechanisms (physiologic and behavioral factors) that link depression to medical illnesses; and management of depression in this group.
\end{abstract}

\section{Introdução}

A s tentativas de compreender a relação entre a depressão e as doenças não-psiquiátricas colocam em evidência todas as dificuldades e as controvérsias que persistem em relação ao humor depressivo, a validade do diagnóstico psiquiátrico e as implicações dos eventos vitais traumáticos para diagnóstico e tratamento dos transtornos psiquiátricos (Rodin et al., 1991).

Doença clínica e depressão são experiências comuns na vida de muitas pessoas. Quando essas doenças coexistem, a depressão tanto pode ser uma complicação de uma doença clínica (ou de seu tratamento), quanto a sua causa, conseqüente a um ou mais fatores etiológicos comuns a ambas, ou, ainda, uma mera coincidência de ocorrência. Em geral é uma relação altamente complexa, algumas vezes mal interpretada e freqüentemente negligenciada.

É importante despertar o interesse do médico clínico para o diagnóstico e o tratamento dos transtornos depressivos, porque sabemos que muitos não identificam, não tratam ou não encaminham pacientes clínicos com depressão para avaliação e tratamento psiquiátricos. No entanto é importante que, ao focalizar nossa atenção sobre depressão, não passemos a impressão de que o estado mental associado à doença clínica é necessariamente sombrio e depressivo. De fato, o que é mais espantoso no contexto de uma doença clínica é a grande capacidade do ser humano em se adaptar a adversidades de todos os tipos. O humor deprimido é uma resposta humana normal ao sentimento de dano, perda, incerteza e vulnerabilidade que pode

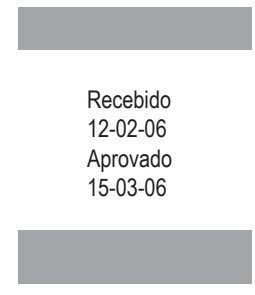

Departamento de Medicina Interna da Universidade Federal de Santa Catarina (UFSC) (Furlanetto LM) Setor de Psicologia Médica do Departamento de Psiquiatria e Medicina Legal da Universidade Federal do Rio de Janeiro (UFRJ) (Brasil MA)

Correspondência para: Letícia Maria Furlanetto Av. Rio Branco, 333 - sala 804 - Centro - 88015-200 - Florianópolis-SC - Tel.: (48) 3223-5594 - Fax: (48) 3234-9756 - e-mail: leticiafurlanetto@yahoo.com.br 
acompanhar as doenças clínicas graves. Na maioria dos casos, os sintomas depressivos não persistem e se remitem assim que há tempo suficiente para a adaptação àquela situação. Contudo o desconforto, o comprometimento da aparência física, a incapacitação e a mudança de vida associados à doença clínica são fatores de risco para depressão, particularmente em indivíduos com história familiar dessa doença (Robinson e Robins, 1989; Rodin et al., 1991; Rundel e Wise, 2005).

Sintomas do tipo depressivo aparecem com freqüência em pacientes com doenças físicas (Polsky et al., 2005). Pesquisas recentes têm mostrado de forma consistente que indivíduos com doenças clínicas que estão deprimidos apresentam menor adesão aos tratamentos propostos (Lustman e Clouse, 2005), menos comportamentos favoráveis de autocuidado (Ziegelstein et al., 2000; Lin et al., 2004), maior prejuízo funcional (Sullivan et al., 1997), diminuição da qualidade de vida (de Jonge et al., 2006b), aumento nos custos (Unutzer et al., 1997) e pior prognóstico, com maiores morbidade (Lesperance et al., 2000) e mortalidade (Furlanetto et al., 2000; Rumsfeld et al., 2005).

O diagnóstico de depressão nos pacientes clínicos é difícil porque vários sinais e sintomas importantes podem ser confundidos com aqueles decorrentes do fato de se ter uma doença física (Furlanetto, 2000). O tratamento da depressão em pacientes com co-morbidade física também é difícil devido a alterações na farmacocinética e na farmacodinâmica decorrentes da condição médica. Existem muitas interações com as medicações usadas para tratar a doença física de base, e há questionamentos quanto à eficácia e à segurança do uso de antidepressivos nesse grupo.

\section{É fácil fazer o diagnóstico de depressão maior num indivíduo hospitalizado por uma doença crônica?}

Dra. Letícia Furlanetto - Freqüentemente temos dificuldades em dizer se um indivíduo hospitalizado por doenças físicas tem depressão maior. Diversas são as causas que podem estar gerando os sintomas do tipo depressivo. Embora tristeza e pessimismo possam indicar uma síndrome depressiva, também podem ser parte de uma adaptação normal a uma doença que ameace a vida. Os sintomas vegetativos e somáticos que fazem parte dos critérios diagnósticos da depressão (fadiga, alterações em sono, peso e psicomotricidade) não são específicos e podem ter como causas hospitalização, tratamentos ou doença de base. Pesquisando pacientes internados em enfermarias de clínica médica, observamos que, entre aqueles sem síndrome depressiva $29 \%$ se queixavam de insônia, $35 \%$ de fadiga e $42 \%$ de perda de peso (Furlanetto, 1996). Apesar disso, foi possível verificar que existem características que confundem menos e que estão presentes de forma significativa nos deprimidos: história prévia de depressão, perda de interesse pelas pessoas e incapacidade de imaginar ter prazer com atividades antes prazerosas (p. ex., comer sua comida predileta, namorar, etc.). Sintomas leves de tristeza, choro, insônia, irritabilidade, inapetência e anedonia são comuns nesse contexto, entretanto só indicam depressão maior quando começam a ter intensidade moderada, persistindo, na maior parte do tempo, de forma autônoma e independente da condição física. Pacientes clínicos, mesmo quando gravemente doentes, ainda conseguem se alegrar quando recebem visitas ou boas notícias sobre sua doença, quando há remissão de dor, náusea ou melhora da condição física (mesmo que discreta). Ajudam bastante as seguintes perguntas: $E$ quando você não tem dor? E se você pudesse comer sua comida predileta? E quando não tem náusea, indisposição? Então, uma boa forma de se começar a avaliar depressão nesse contexto é verificar história pessoal e familiar de depressão, assim como se houve diminuição, pelo menos moderada, da capacidade de imaginar ter prazer (com visitas, comida predileta, libido) (Gauer et al., 2003).

Outra forma de se abordar o problema é verificar quando a síndrome apresentada já tem significado clínico. Ou seja, quando já leva a alteração no prognóstico esperado para aquela doença de base. Nesse sentido verificamos, em outra pesquisa, que pacientes com história prévia de depressão, indecisão, insônia, baixa auto-estima, desesperança ou anedonia morriam significativamente mais durante a internação, independentemente da gravidade física inicial (Furlanetto et al., 2000; von Ammon Cavanaugh et al., 2001).

0 reconhecimento da depressão durante a internação é fundamental, tanto porque está associada a pior prognóstico, como porque já se notou que, caso um deprimido não seja medicado durante a internação, a chance de isso ocorrer depois é muito pequena (Koenig et al., 1997a).

\section{Existem outros diagnósticos do grupo dos transtornos depressivos que se aplicariam melhor ao ambiente hospitalar?}

Dr. Marco Antonio Brasil - No contexto de uma doença clínica, o médico tem que diferenciar sintomas de depressão maior não só daqueles de transtorno de ajustamento e de reação normal à doença, mas também daqueles que são manifestação direta da própria doença clínica.

A avaliação de depressão é difícil devido à fronteira imprecisa e, às vezes, arbitrária entre as formas clínicas, subclínicas e as não-patológicas. Essa dificuldade atinge o seu ápice quando se trata de um paciente com doença clínica. Nesse caso, essas distinções se tornam ainda mais problemáticas, posto que há uma base real para o sentimento de tristeza associado a uma doença clínica grave, e porque sintomas somáticos como anorexia ou perda de energia podem ser decorrentes da doença clínica. No caso, por exemplo, de um paciente diabético, já quase cego e com insuficiência renal, muitas vezes é difícil saber se o seu pessimismo acerca do futuro é uma resposta realista a sua situação de vida ou uma manifestação de depressão.

A diferenciação entre depressão e tristeza no contexto de uma doença clínica é geralmente muito complicada, mas alguns sintomas servem de orientação. Na depressão, costuma haver sentimentos de culpa e auto-acusação, indiferença generalizada pelo ambiente e, nos casos mais graves, ideação suicida. A tristeza geralmente não é tão prolongada quanto a depressão, costumando ter uma duração inferior a seis meses, e os sintomas de culpa e auto-acusação e ideação suicida não estão presentes. O paciente com apenas tristeza é geralmente reativo ao ambiente, podendo responder de modo favorável aos estímulos externos (por ex., visitas de familiares, do médico assistente, boas notícias a respeito de sua recuperação). 
O transtorno de ajustamento situa-se na fronteira entre 0 normal e o patológico. A reação normal a mudanças estressantes (aparecimento de uma doença grave, por exemplo) é um breve período de ansiedade e depressão, às vezes com irritabilidade e pouca concentração. Pode haver um pequeno tempo de negação, logo seguido pela resolução do problema. $O$ transtorno de ajustamento ocorre quando a resposta a uma situação de estresse é considerada anormal, e isso acontece quando ela é maior do que a esperada para aquele determinado evento; é acompanhada de comprometimento funcional e/ou social; e quando a reação dura um período limitado e não é grave o suficiente para ser diagnosticada como outro transtorno psiquiátrico.

No critério para episódio depressivo maior do Manual Diagnóstico e Estatístico de Transtornos Mentais (DSM-IV), exige-se que cinco (ou mais) de nove sintomas listados tenham estado presentes por pelo menos um mesmo período de duas semanas e que representem um comprometimento da capacidade funcional anterior, sendo que pelo menos um dos sintomas presentes seja o humor deprimido ou a perda do interesse ou prazer. Dos sintomas listados pelo DSM-IV, seis podem estar presentes nos pacientes com doença clínica como expressão da própria doença (humor deprimido, alterações do apetite e do sono, agitação ou retardo motor, fadiga e dificuldade de concentração).

Embora seja importante fazer a distinção entre transtorno depressivo e sintomas depressivos, acredita-se que há alguma similaridade, se não um continuum, entre as depressões clínica e subclínica. Se nos limitássemos exclusivamente aos transtornos psiquiátricos dentro dos critérios definidos, teríamos que excluir estados subclínicos de disforia e estresse vivenciados por pacientes que se beneficiariam com uma intervenção terapêutica.

Entendemos que há necessidade de um novo tipo de classificação psiquiátrica orientado para situações específicas de hospitais gerais. Uma classificação e um conjunto de instrumentos diagnósticos que foram originados em pesquisa com pacientes psiquiátricos nem sempre são apropriados para pacientes de hospital geral (HG) (Rodin et al., 1991; Brasil e Furlanetto, 1997; Strain, 2005).

Neste contexto de HG e atenção primária é importante lembrarmos da observação arguta de Goldberg e Huxley (1992): "Ao invés de uma miríade de subdivisões dos transtornos mentais menores encontrados nas classificações CID e DSM (...) nós afirmamos que há somente um número limitado de maneiras que 0 ser humano responde ao estresse psicológico, e que são definidas por duas dimensões subjacentes de sintomatologia: sintomas ansiosos por um lado e sintomas depressivos por outro. Estas duas dimensões estão relacionadas entre si e a combinação destes dois grupos é mais comum do que cada grupo em separado".

\section{Qual é sua opinião sobre as tentativas de se utilizarem critérios diagnósticos específicos para pacientes com depressão e doenças clínicas?}

Dra. Letícia Furlanetto - Sempre houve muita preocupação quanto à utilização das classificações atuais (DSM-IV e Classificação Internacional de Doenças [CID-10]) em pacientes com doenças físicas. Grande parte dos estudos que geraram esses manuais foi feita em serviços de psiquiatria (hospitais e ambulatórios especializados), onde são encontrados casos psiquiátricos mais graves. Além disso, aqueles indivíduos com doenças físicas eram excluídos. Na atenção primária e nos hospitais gerais, onde os pacientes com doenças físicas são atendidos, o que encontramos são casos mais leves e mistos (Brasil e Furlanetto, 1997). Além disso, existe a preocupação de que, usando-se as classificações atuais, muitos indivíduos sem depressão seriam classificados como depressivos, quando na realidade os sintomas que geraram o diagnóstico (alterações no apetite ou peso, sono, fadiga e dificuldade de concentração) poderiam ter sido causados somente pela doença de base, por medicamentos ou pela internação. Por isso alguns autores procuraram elaborar critérios diagnósticos específicos para depressão em pacientes com doenças físicas. Endicott (1994) sugeriu a substituição de sintomas que poderiam se confundir com os causados pelo câncer. Dessa maneira, alterações de apetite/peso, sono, energia e concentração seriam substituídos por aparência depressiva, retraimento social (perda do interesse pelas pessoas), pessimismo e humor não-reativo, respectivamente (Endicott, 1984). Chochinov et al. avaliaram a validade da substituição proposta por Endicott e verificaram que, quando os pacientes apresentavam humor depressivo ou anedonia de intensidade pelo menos moderada (na maior parte do tempo), não havia diferença caso se considerassem esses sintomas somáticos ou se fosse feita a substituição proposta (Chochinov et al., 1994). Já Koenig et al. verificaram que, quando os sintomas somáticos só foram computados porque não puderam ser explicados pela condição médica, em cerca de $50 \%$ dos pacientes a depressão maior persistente não foi detectada (Koenig et al., 1997b).

Em pesquisa recente, Simon e Von Korff elucidaram a questão da validade dos sintomas somáticos para os critérios de depressão do DSM-IV (Simon e Von Korff, 2006). Com um estudo longitudinal acompanhando quase 500 deprimidos com e sem co-morbidade física durante seis meses, observaram que os sintomas somáticos (fadiga, alterações de peso ou apetite, sono ou psicomotricidade) foram válidos para o diagnóstico e melhoraram com o tratamento antidepressivo da mesma maneira nos pacientes sem e com doenças físicas crônicas (Simon e Von Korff, 2006).

Resumindo, as pesquisas citadas não mostraram benefício de critérios específicos nesse grupo. Na minha opinião, só não devemos computar sinais e sintomas quando eles puderem ser facilmente explicados pela doença de base ou hospitalização (p. ex., o paciente explica que só não dorme devido ao barulho no quarto). Uma abordagem muito útil no dia-a-dia parece ser a proposta por Cavanaugh, na qual sintomas como fadiga e alterações de sono, apetite, peso e psicomotoras ajudam a corroborar o diagnóstico de depressão quando em excesso ao esperado para a condição física e seus tratamentos e quando surgem associados temporalmente aos sintomas cognitivo-afetivos da depressão (humor depressivo, anedonia) (von Ammon Cavanaugh, 1995)

\section{Fale um pouco sobre o conceito de desmoralização e sua relevância para o profissional que trabalha no HG.}

Dr. Marco Antonio Brasil - O termo encontrado na literatura norte-americana denominado demoralization poderia ser traduzido, com reservas, para o português como desmoralização, 
no sentido de perda da força moral, do comprometimento, da autoconfiança, da coragem e da capacidade de decisão. Desmoralização é um construto psicológico e psicossocial que permeia transtornos mentais específicos, compartilhando algumas características de depressão maior, transtorno de somatização e de ansiedade (Brasil, 1995).

Os sintomas de transtorno de ansiedade generalizada não se superpõem claramente aos de desmoralização. Os sintomas principais da desmoralização, como baixa auto-estima e tristeza, não são usualmente encontrados em pessoas com transtorno de ansiedade generalizada, embora o medo e a ansiedade, presentes na desmoralização, também o estejam no transtorno de ansiedade generalizada. Assim, mesmo que a ansiedade generalizada seja meramente uma expressão de estresse psicológico e psicossomático não-específico, ela tem um critério mais restritivo do que a desmoralização (Blazer et al., 1993).

A maior superposição e a dificuldade de diferenciação estão entre a desmoralização e a depressão. Mangelli et al. (2005), estudando uma população de pacientes com doenças somáticas, identificaram desmoralização em 245 pacientes $(30,4 \%)$ e depressão maior em $135(16,7 \%)$. Apesar de ter havido uma superposição considerável entre os dois diagnósticos, 59 pacientes $(43,7 \%)$ com depressão maior não foram classificados como tendo desmoralização e 169 indivíduos (69\%) com desmoralização não cumpriram os critérios para depressão maior. Esses achados de Mangelli et al. (2005) sugerem uma alta prevalência de desmoralização entre pacientes com doenças somáticas e a possibilidade de diferenciação entre depressão e desmoralização.

Schildkraut e Klein (1976) definiram desmoralização como um sentimento de incapacidade após repetidas derrotas e uma subseqüente sensação de desamparo. Esses autores consideram 0 aspecto principal para diferenciar desmoralização da depressão $o$ fato de que as pessoas deprimidas perdem a capacidade de sentir prazer (anedonia), enquanto as com desmoralização apresentam um sentimento de incapacidade e de perda do autodomínio.

Frank e Figueiredo $(1982,1983,1993)$ também procuram diferenciar desmoralização de depressão. A desmoralização seria uma combinação de estresse e uma impressão subjetiva de incompetência, resultando numa incerteza sobre que atitude tomar, enquanto que na depressão haveria diminuição da motivação mesmo quando uma direção de ação apropriada é conhecida. Um contraste semelhante foi observado por Clarke e Kissane (2002), que viram a desmoralização como um estado de desespero existencial, desamparo, desesperança e perda do sentido e do propósito da vida, acompanhados de um sentimento subjetivo de incompetência, enquanto que a anedonia seria o sintoma nuclear da depressão. Shader (2005) observa, com base em sua experiência clínica, que quem está com desmoralização pode responder favoravelmente a um estímulo positivo e ao alívio dos seus estressores; já o deprimido não pode se livrar de seu estado negativo de humor, permanecendo independente das mudanças ambientais.

O contexto da dor crônica é favorável ao aparecimento da desmoralização, pois muitos pacientes nesse caso sentem-se desamparados, desesperançados e sem iniciativa no sentido de não saber o que fazer para se ajudarem ou serem ajudados. A melhora do sentimento de autocontrole e a redução da sensação de desamparo freqüentemente podem ser facilitadas por um envolvimento mais ativo do paciente nas decisões acerca de tratamento e de uso maior de analgesia. Uma boa e detalhada avaliação clínica é necessária para determinar quando usar antidepressivos (tanto pelo seu valor como antidepressivo como pelo seu efeito sobre o circuito da dor), quando e quanto de analgesia deve ser usada (e por que via de administração e liberação) e o que fazer para ajudar os pacientes a se sentirem mais esperançosos e com maior segurança e domínio sobre a dor.

Shader (2005) observa que, na sua experiência clínica, a desmoralização per se geralmente não responde aos antidepressivos, embora agentes dopaminérgicos possam ocasionalmente ser úteis. Os clínicos, uma vez que reconheçam a presença de desmoralização, devem trabalhar com o paciente para promover o sentimento de capacidade, domínio (mastery) e o retorno da esperança. Encorajamento, suporte e educação são essenciais. Esforços para restabelecer o sentimento de capacidade devem ser empregados e ser consistentes com 0 nível de compreensão e as atitudes do paciente.

Griffith e Gaby (2005) salientam o papel da psicoterapia breve (bedside psychotherapy) em pacientes com doenças somáticas não-psiquiátricas para lidar com a desmoralização, que seria o desespero, o sentimento de desamparo e o isolamento que muitos pacientes enfrentam quando afetados por suas doenças e seus tratamentos. A desmoralização poderia ser vista como um conjunto de diferentes posturas existenciais que fazem o paciente renunciar a enfrentar os desafios trazidos pela doença. Uma estratégia proposta por esses autores é tentar identificar quais os temas existenciais que mais o preocupam e então elaborar questões e intervenções para tratar especificamente desses temas. Tais intervenções ajudariam os pacientes a lidar com a doença de maneira mais assertiva, mobilizando posturas existenciais de resistência, como esperança, força e maior comunicação com as outras pessoas.

Como afirma Shader (2005), a literatura atual sobre a desmoralização é, na melhor das hipóteses, esparsa. Serão necessárias novas pesquisas no sentido de melhor avaliar quais as implicações e a importância do conceito de desmoralização para a prática clínica. Sua importância como fator de risco para depressão (Shader, 2005), na evolução e no prognóstico de doenças somáticas não-psiquiátricas (Rafanelli et al., 2005) deverá ser confirmada em estudos longitudinais.

\section{Qual é o real papel da doença clínica na fisiopatologia da depressão? Existem novas teorias sobre causalidade nessa área?}

Dra. Letícia Furlanetto - Durante algum tempo havia o questionamento quanto a se a depressão em pacientes com doenças físicas seria devida ao processo de adoecer. De acordo com essa visão, os sintomas depressivos seriam uma mera reação à doença física e se remitiriam espontaneamente com o tempo. Contudo o que se observa é que, em pacientes com síndrome depressiva moderada a grave, não só os sintomas são persistentes (de Jonge et al., 2006a) e não remitem 
espontaneamente (Furlanetto et al., 1998), como também, mesmo após o tratamento antidepressivo, eles ainda morrem mais que os não-deprimidos, independente da gravidade física inicial (Lesperance et al., 2002). O que se nota, na realidade, é que a maior parte dos deprimidos admitidos no HG já chega com sintomas depressivos (Furlanetto et al., 1998). Uma explicação seria que indivíduos predispostos (com antecedentes pessoais e familiares de depressão), diante dos estressores (doenças físicas, medicamentos, hospitalização, dor, perdas e medo da morte), estariam mais vulneráveis a sofrer um agravamento de sintomas depressivos residuais ou mesmo a desenvolver um novo episódio.

Por outro lado, existem indivíduos idosos que nunca tiveram depressão ao longo da vida e que só mais tarde desenvolvem uma síndrome depressiva (depressão tardia). Nesse grupo que tem menor carga genética (pouca história familiar) para depressão e no qual a psicopatologia costuma ser mais branda, têm sido reveladas alterações cerebrais que sugerem anormalidades fisiológicas na substância branca (Kumar et al., 2002; Nobuhara et al., 2006). Nesse mesmo sentido, trabalhos avaliando pacientes que tiveram depressão após um acidente vascular encefálico (AVE) associam seu aparecimento a lesões neuroanatômicas, embora haja controvérsia quanto à localização das lesões serem significativamente mais freqüentes no lobo frontal esquerdo (Robinson, 2003) ou se não faria diferença a localização do AVE para seu aparecimento (Carson et al., 2000; Gainotti et al., 2001). Nesse caso, poderíamos chamar essa depressão de secundária (apareceu depois da doença física). Outras doenças também estão associadas ao aparecimento de depressão, provavelmente devido também a processos neurodegenerativos, como as doenças de Alzheimer (Zubenko et al., 2003) e de Parkinson (Mossner et al., 2001). Além disso, quando há liberação de fator de necrose tumoral (TNF) (como no câncer) ou de outras citocinas pró-inflamatórias (p. ex., pelo tecido adiposo e muscular de obesos diabéticos) (Musselman et al., 2003), o indivíduo pode apresentar indisposição e comportamento de doente, que é semelhante fenomenologicamente à síndrome depressiva (Schiepers et al., 2005; Simen et al., 2006). A ativação do sistema imune (aumento de interleucina 2 [IL2] e IL6), devido ao uso de interferon em pacientes com hepatite C, também foi associada ao aparecimento e à gravidade da síndrome depressiva (Wichers et al., 2006).

Exceto nos casos citados acima, não é tão fácil fazer essa associação linear entre doenças físicas e surgimento posterior de depressão. Revisando a literatura encontramos resultados controversos. Três estudos longitudinais recentes mostraram essa associação em pacientes com câncer, doença pulmonar obstrutiva crônica (DPOC), artrite reumatóide (AR), infarto agudo do miocárdio (IAM), insuficiência cardíaca congestiva (ICC), lesão em extremidades por quedas e obesidade (Roberts et al., 2003; Polsky et al., 2005; de Jonge et al., 2006a). Contudo é possível que os indivíduos já tivessem sintomas depressivos subclínicos não-diagnosticados na primeira avaliação. Além disso, também poderia existir alguma disfunção ou história familiar que predisporia tanto à doença física quanto à depressão (Appolinario et al., 2004).

0 reconhecimento da depressão como um fator que leva a maior morbimortalidade já vem sendo relatado há algum tempo, sobretudo em pacientes após IAM (Frasure-Smith et al., 1993; Lesperance et al., 1996). Esse efeito deletério da depressão também tem sido demonstrado em outras patologias, como diabetes (de Groot et al., 2001; Lustman e Clouse, 2005), AVE (House et al., 2001), vírus humano da imunodeficiência (HIV)/síndrome da imunodeficiência adquirida (AIDS) (Leserman et al., 2002), câncer (Spiegel e Giese-Davis, 2003), e em pacientes internados em enfermarias gerais (von Ammon Cavanaugh et al., 2001).

0 mais intrigante tem sido a constatação de que sintomas depressivos podem levar ao aparecimento posterior de doenças físicas, independentemente de fatores de risco, em patologias como hipertensão arterial (Everson et al., 2000), IAM (Pratt et al., 1996), diabetes tipo 2 (Golden et al., 2004), AVE (Larson et al., 2001), epilepsia (Hesdorffer et al., 2000) e doença de Alzheimer (Green et al., 2003).

Os mecanismos que explicam estas alterações têm aspectos comportamentais (por baixa adesão a comportamentos de risco, etc.) (Wang et al., 2002), mas também existem fatores fisiológicos que vêm sendo implicados nessa associação, como hiperatividade do eixo hipotálamo-hipófise-adrenal (EHHA) (Ehlert et al., 2001), diminuição da variabilidade da freqüência cardíaca (Kuo et al., 2005), aumento dos fatores de coagulação (Kop et al., 2002), ativação de sistemas neuroimunológicos (Kop e Gottdiener, 2005), ativação da agregação plaquetária (Laghrissi-Thode et al., 1997) e aumento na expressão dos marcadores inflamatórios (Miller et al., 2002), os quais ocorrem na depressão.

Parece haver um impacto bidirecional na co-morbidade de depressão e doenças físicas. Novas teorias sobre a fisiopatologia da depressão advogam que esta seria devida a mudanças no volume do hipocampo (Eisch e Nestler, 2002). 0 estresse mantido aumentaria a liberação constante de cortisol, o que levaria à diminuição do hipocampo, que, por sua vez, deixaria de fazer feedback negativo em nível de EHHA, aumentando ainda mais a liberação desse hormônio (Manji, 2003). Outros fatores também afetam a neuroplasticidade cerebral, como insuficiência cerebrovascular (como visto nas doenças cardíacas, AVE, diabetes, etc.), episódios repetidos de depressão, fatores genéticos e alterações no fator neurotrófico derivado do cérebro (BDNF, que se reduz devido a estresse, depleção de serotonina ou norepinefrina e aumenta com o tratamento antidepressivo) (Manji, 2003).

Resumindo, há uma via de mão dupla na qual tanto a depressão pode levar a comportamentos desfavoráveis e a alterações fisiológicas que precipitam doenças clínicas, quanto as doenças físicas e seus tratamentos são fatores estressantes e causam alterações fisiológicas que podem precipitar ou agravar a depressão (Evans et al., 2005; Lustman e Clouse, 2005).

\section{Qual é a epidemiologia da depressão hospitalar e em ambulatórios especializados? E na assistência primária? Quais são as semelhanças e as diferenças entre os dados internacionais e nacionais?}

Dra. Letícia Furlanetto - Estudos que avaliam depressão em pacientes com doenças físicas mostram taxas de $3 \%$ a 
$55 \%$ (Evans et al., 2005). Essa ampla variação provavelmente decorre de aspectos metodológicos (instrumentos utilizados, definição de caso, local, etc.). Assim, por exemplo, em uma mesma amostra de pacientes internados em enfermarias gerais, podem-se obter as seguintes freqüências: $6 \%$ a $12 \%$, caso se usem os critérios para transtorno depressivo (CID-10 ou DSM); cerca de $20 \%$, caso se pesquisem sintomas depressivos significativos (provavelmente englobando aqueles indivíduos que têm história de depressão mas não atingem o limiar dos critérios no momento da avaliação); e 30\% ou mais, dependendo da escala e do ponto de corte utilizado (aqui são incluídos também indivíduos sem história pessoal e familiar para depressão e que podem ter remissão espontânea, mas que, como grupo, ainda podem ter pior evolução do que aqueles sem sintomas depressivos) (Furlanetto et al., 1998). Se esses mesmos pacientes fossem avaliados com os mesmos instrumentos, com sua doença física de base compensada, nos ambulatórios especializados, provavelmente a prevalência seria mais baixa.

Aqui no Brasil, os resultados são semelhantes aos encontrados nos estudos internacionais. Pacientes internados em hospitais gerais tiveram taxas que variaram de $19 \%$ a $50 \%$ (Nascimento e Noal, 1992; Botega et al., 1995; Furlanetto, 1996; Kapczinski et al., 1996; Furlanetto et al., 1998; Morimoto et al., 1998; Fornari e Furlanetto, 2002). Já no pronto-socorro geral foi encontrada prevalência de depressão de cerca de 11\% (Shirakawa e Jorge, 1983; Fleck e Soares, 1990). Em ambulatórios de $\mathrm{HG}$ a freqüência de transtornos depressivos recorrentes foi de 15,8\%, e de distimia, 2,4\% (Villano et al., 1995). No grupo de pacientes com transtornos mentais comuns atendidos nas unidades do Programa de Saúde da Família (PSF), a porcentagem de transtornos depressivos atuais foi de $30,7 \%$ (Fortes, 2004).

\section{Quando se avalia a capacidade diagnóstica de depressão dos profissionais que trabalham em hospitais clínicos, geralmente temos um retrato não muito animador. Quais são as perspectivas para lidar com esse problema? O que se tem feito no mundo e no Brasil a esse respeito?}

Dra. Letícia Furlanetto - Realmente existem problemas no reconhecimento da depressão em pacientes clínicos. Muitos pacientes deprimidos deixam de ser detectados porque não falam seus sintomas para o médico com medo de serem estigmatizados (Furlanetto, 1997a; Jamison, 2006). Além disso, por questões de tempo limitado, assim como falta de treinamento e de conhecimento anterior do indivíduo, a depressão pode não ser reconhecida nos pacientes clínicos (Montano, 1994). Os médicos, por sua vez, devido à doença física, muitas vezes consideram normal qualquer manifestação de sofrimento.

Tão importante quanto o problema da não-detecção de deprimidos vem sendo a prescrição de antidepressivos e benzodiazepínicos para pacientes sem depressão. Existe 0 risco de diagnosticar vários pacientes clínicos como tendo depressão mascarada quando não se encontra prontamente uma causa orgânica para a queixa relatada (Furlanetto, 1997b). Um estudo epidemiológico recente revelou que somente metade dos pacientes que recebiam psicotrópicos preenchia critérios para transtornos mentais (Kessler et al., 2005). No HG existe um excesso de diagnóstico de depressão em pacientes que apresentam na realidade delirium decorrente da condição médica geral e de medicamentos (Farrell e Ganzini, 1995). Pacientes com síndrome confusional leve podem se queixar de que não dormem e ter labilidade emocional, com choro fácil e disforia, que são confundidos, pelo médico, com depressão ou ansiedade.

Apesar de existirem escalas para rastreamento da depressão no HG, que inclusive foram validadas em amostras brasileiras (Botega et al., 1995; Furlanetto et al., 2005; Fráguas et al., 2006), há que se lembrar de que elas não substituem uma avaliação clínica criteriosa e que existe o risco de se começar a medicar o papel em vez do sujeito. Por isso elas são muito úteis para o ensino e a pesquisa, mas não sugiro que sejam usadas no dia-a-dia.

Além disso, o que se observa é que o problema não está somente na detecção da depressão. Mesmo quando os médicos assistentes são avisados de que seus pacientes estão deprimidos, o tratamento muitas vezes não ocorre ou é inadequado (Cigognini e Furlanetto, 2006). Hoje em dia está-se dando mais valor ao treinamento de médicos generalistas não só para 0 reconhecimento da depressão, mas, sobretudo, para o melhor manejo de pacientes deprimidos como forma de aprimorar a qualidade do atendimento (Wells et al., 2005).

\section{O tratamento da depressão no paciente clínico se diferencia daquele do paciente com depressão sem doença clínica?}

Dr. Marco Antonio Brasil - Os pacientes clínicos, ou seja, aqueles com uma co-morbidade de depressão e uma doença orgânica (não-psiquiátrica), apresentam uma série de fatores que dificultam o diagnóstico e o tratamento da depressão. São pacientes com idade média superior à daqueles sem doença clínica, geralmente em uso de mais medicamentos, padecendo de maior número de sintomas (decorrentes da depressão e da doença clínica) e submetidos às alterações na farmacocinética e na farmacodinâmica decorrentes da doença clínica. Isso traz como conseqüência problemas de interação medicamentosa, agravamento e somação de efeitos colaterais, maior comprometimento funcional, além de prejudicar a adesão ao tratamento. Ademais, o paciente que apresenta co-morbidade, depressão e doença clínica pode estar sujeito a maior gasto com o tratamento (medicamentos, exames), maior número de visitas ao médico e submissão a exames invasivos e dolorosos. Duas questões se destacam em relação ao tratamento da depressão em pacientes com doença clínica: quando e como tratar estes pacientes.

Para aqueles com diagnóstico de depressão maior, o tratamento principal será o farmacológico com antidepressivos. No entanto os ensaios terapêuticos controlados com medicamentos antidepressivos nessa população são ainda muito poucos e raramente estabelecem comparações sobre a eficácia relativa entre as diferentes modalidades de tratamento. Nesses pacientes com doença clínica, sobretudo nos idosos, deve-se iniciar com doses baixas e aumentar lenta e cuidadosamente, observando atentamente a tolerância ao novo medicamento introduzido. Lembremo-nos de que ainda conhecemos muito 
pouco sobre os efeitos dos antidepressivos, sobretudo em longo prazo, nessa população de pacientes. Por outro lado, é praticamente impossível para o psiquiatra saber todas as possíveis interações entre antidepressivos e medicamentos clínicos. Sendo assim, é recomendável que se tenha sempre à mão um livro de referência sobre efeitos colaterais e interações medicamentosas. Devemos também ter em mente que o transtorno depressivo pode ser secundário a uma doença clínica (hipotireoidismo e doença de Cushing, p. ex.) ou a um medicamento clínico (corticóide e interferon, p. ex.) (Rundell e Wise, 2005; Brasil, 2001).

Nos pacientes com transtorno de ajustamento com depressão, a intervenção psicoterápica é o principal recurso, com prioridade para terapia de grupo, com indivíduos com a mesma patologia clínica ou orientação sobre a doença pelo médico assistente.

Em relação a quem deve ser tratado, depende de uma avaliação diagnóstica. A rigor, todo o paciente que receba 0 diagnóstico de depressão maior, distimia, transtorno de ajustamento com depressão e transtorno orgânico do humor deve receber tratamento.

\section{Qual é o papel do tratamento farmacológico da depressão no paciente clínico? Quais são os agentes mais estudados?}

Dra. Letícia Furlanetto - Uma vez havendo a indicação de antidepressivos, o seu uso pode ajudar não só na remissão da depressão, mas também na diminuição das suas morbidade e mortalidade. Portanto, juntamente com outras medidas (redução ou troca, quando possível, de medicações depressogênicas, manejo da doença física e suporte), o papel dos antidepressivos é fundamental, porque, de acordo com pesquisas recentes, quando cautelosamente prescritos, podem melhorar de forma significativa o prognóstico clínico (Lustman e Clouse, 2005; Sherwood et al., 2005; Taylor et al., 2005).

No passado, a medicação mais estudada em pacientes com doenças físicas e em idosos foi a nortriptilina, devido ao fato de apresentar menos hipotensão postural e menos efeitos anticolinérgicos do que os outros tricíclicos (Andersen et al., 1980; Lipsey et al., 1984). Apesar da preocupação quanto aos seus efeitos quinidiniformes, que podem precipitar arritmias cardíacas, além do risco aumentado de síndrome confusional e de ser hiperglicemiante (Lustman et al., 1997), ainda é o tricíclico mais estudado para pacientes clínicos (Robinson et al., 2000). Pode ser útil naqueles pacientes que não possuem contra-indicação, já que tem efeitos sedativos, aumenta o apetite e também é analgésica. Para pacientes com dor com ou sem depressão as medicações que mostraram eficácia de forma consistente foram os tricíclicos e a venlafaxina (Fishbain, 2000).

Atualmente, as medicações mais promissoras são os inibidores seletivos da recaptação da serotonina (ISRS) e, mais especificamente, a sertralina e o citalopram (e potencialmente o escitalopram) por terem menor risco de interações medicamentosas (Andersen et al., 1994; Glassman et al., 2002; Taylor et al., 2005). Outros ISRS (embora tenham mostrado eficácia nesse grupo, como no caso da paroxetina e da fluoxetina) apresentam maior risco quando associados a medicamentos clínicos, podendo levar a desfechos desfavoráveis (Yasui-Furukori, Kaneko, 2006). A venlafaxina, que é um antidepressivo dual, foi testada em pacientes com dor e tem pouca interação medicamentosa, mas é vista com cautela devido ao risco de aumentar a pressão arterial (Degner et al., 2004). A erva-de-sãojoão deveria ser evitada em pacientes com doenças físicas por levar a alterações na farmacocinética de diversos medicamentos (Zhou et al., 2004).

Infelizmente ainda não temos muitos estudos controlados nessa população. Existem alguns estudos abertos e muitos artigos sugerindo que tais e tais medicações são seguras e eficazes, mas, na maioria dos casos, sem nenhum ensaio clínico controlado comprovando suas afirmações. Fico me perguntando se isso seria decorrente do viés dos estudos negativos (pesquisas que não foram favoráveis e, por isso, não foram publicadas).

A especialidade mais desenvolvida nesse sentido é a cardiologia, com duas pesquisas bem controladas, em amostras significativas de pacientes deprimidos avaliados após sofrerem IAM, que mostraram que a sertralina foi segura e eficaz (Glassman et al., 2002; Taylor et al., 2005). Estudos controlados mostraram a eficácia antidepressiva também em pacientes com as seguintes doenças: 1) AVE - com a nortriptilina e o citalopram. A fluoxetina teve resultados controversos nesse grupo (Andersen et al., 1994; Gonzalez-Torrecillas et al., 1995; Wiart et al., 2000; Robinson, 2003); 2) diabetes mellitus - com a nortriptilina (embora tenha sido hiperglicemiante nos diabéticos não-deprimidos) (Lustman et al., 1997) e a fluoxetina (Lustman et al., 2000), a melhora do nível glicêmico também ajudou a reduzir os sintomas depressivos em diabéticos (Lustman, Clouse, 2005); 3) no câncer - com desipramina, fluoxetina, paroxetina e amitriptilina (Holland et al., 1998; Pezzella et al., 2001); 4) AIDS - com a fluoxetina e a sertralina (Ferrando et al., 1999; Rabkin et al., 1999); 5) doença de Alzheimer - com a fluoxetina e a sertralina (Petracca et al., 2001; Lyketsos et al., 2003).

Apesar da sugestão do uso preventivo de antidepressivos em pacientes que iam ser medicados com interferon (Musselman et al., 2001), não há evidências conclusivas que comprovem maior benefício dessa prática nos pacientes clínicos. Mesmo as medicações mais testadas e seguras, como os ISRS, ainda estão associadas a inúmeros efeitos indesejáveis, como hemorragia digestiva alta (Dalton et al., 2003), aumento de enzimas hepáticas (Degner et al., 2004), síndrome da secreção inapropriada do hormônio antidiurético, levando a hiponatremia (Arinzon et al., 2002), disfunções sexuais (Montejo et al., 2001) e alterações neurológicas (Degner et al., 2004), entre outros.

Como já dito anteriormente, a maior parte dos deprimidos já chega ao HG com sintomas depressivos e tem história prévia dessa doença. Por isso me parece mais adequado sempre perguntar sobre história prévia de depressão e ficar atento aos sintomas citados acima para iniciar o tratamento precoce, quando indicado. Além disso, o manejo do paciente clínico deprimido envolve uma série de medidas e não só a medicação antidepressiva. Portanto mais importante do que iniciar um antidepressivo sem muita convicção ou achar que ele sozinho é suficiente é fazer a seguinte pergunta: com quais medidas este paciente pode se beneficiar? 


\section{Qual é o papel da psicoterapia nesse grupo de pacientes? Existe uma modalidade de psicoterapia mais estudada?}

Dr. Marco Antonio Brasil - Os tratamentos psicoterápicos podem ser úteis tanto como terapêutica complementar quanto como principal recurso terapêutico. É importante que na abordagem psicoterápica sejam avaliados os diversos fatores de estresse e de história pessoal que podem estar envolvidos no aparecimento do quadro depressivo.

Embora haja várias abordagens terapêuticas, a literatura científica praticamente tem se limitado a trabalhos com psicoterapia cognitivo-comportamental. Têm surgido trabalhos que apontam para a utilidade e a efetividade desse tipo de psicoterapia no tratamento da depressão em pacientes com doenças clínicas.

O estudo de Lustman (2005), de dez semanas de terapia cognitivo-comportamental juntamente com educação de apoio sobre diabetes, mostrou ser efetivo no tratamento de pacientes deprimidos com diabetes não-insulinodependentes, tendo notado também melhora do controle hipoglicêmico. Kunik (2001) mostrou que o uso breve da terapia cognitivo-comportamental levou a uma redução dos sintomas depressivos, ainda que sem melhora do funcionamento físico, em pacientes com DPOC. No estudo prospectivo feito por Leibing (1999), a terapia cognitivo-comportamental teve um impacto positivo nos níveis de depressão em pacientes ambulatoriais com artrite reumatóide. Têm sido também descritos vários efeitos benéficos das intervenções psicossociais na depressão de pacientes oncológicos. Bloch (2000) descreveu algumas dessas intervenções em psicooncologia. White publicou em 2001 um guia para a aplicação de terapia cognitiva comportamental no tratamento de doenças crônicas.

Será muito importante que trabalhos utilizando outras técnicas psicoterápicas - psicodinâmica, sistêmica, psicodrama, etc. - sejam avaliados nessa população de pacientes. A pobreza de estudos em psicoterapia denuncia uma carência importante na formação do psiquiatra atual.

O tratamento farmacológico deve ser sempre considerado quando os sintomas de depressão persistirem apesar do tratamento da doença clínica subjacente e/ou psicoterápico.

\section{Conclusão}

Apesar das dificuldades diagnósticas ao avaliar depressão nos pacientes com doenças físicas, existem dicas para auxiliar na deteç̧ão, como, por exemplo, perguntar sobre história prévia e se o indivíduo ainda consegue imaginar ter prazer (com visitas, comida predileta, etc.). Nesse sentido, estudos recentes reafirmam a importância de não abandonarmos os sintomas somáticos como critérios para depressão nesse grupo.

Existem fatores comportamentais e fisiológicos implicados na co-morbidade entre depressão e doenças físicas. A depressão aparece como fator de risco independente, levando ao aparecimento de algumas doenças físicas. Por outro lado, pacientes com doenças físicas acabam tendo mais depressão. Na maior parte das vezes, os pacientes aparecem no $\mathrm{HG}$ com sintomas depressivos, doenças físicas e relato de fatores estressantes. Há um impacto bidirecional, no qual, através de uma via de mão dupla, uma agrava a outra. Além disso, ambas (depressão e doença física) são precipitadas por e geram fatores estressantes.

A depressão nos pacientes clínicos é tratável. Existem estudos controlados com antidepressivos para determinadas doenças, sendo os mais estudados, no momento, os ISRS. Há que se ter cautela ao utilizar antidepressivos nesse grupo e saber que o tratamento não se restringe à prescrição de medicamentos. Mais importante do que a prescrição automática dessas substâncias para os pacientes que preenchem critérios é a avaliação judiciosa de quais medidas podem trazer benefícios para cada um em particular.

0 psiquiatra que é treinado para apenas diagnosticar doença está fadado a só ver a doença e perder as nuanças e dramas da experiência humana. Tal abordagem limitada tende a enfatizar mais as doenças do que os pacientes que dela padecem. Sob tal perspectiva, a tristeza que se associa à perda da saúde, com comprometimento da qualidade de vida, pode ser vista pelo médico apenas como uma depressão a ser medicada, o que inevitavelmente desumaniza o paciente. Por outro lado, uma abordagem humanística que ignora a presença de doenças psiquiátricas tratáveis, por compreendê-las psicologicamente, pode privar o paciente do alívio que pode advir de intervenções efetivas e específicas. Infelizmente, depressões clinicamente significativas são freqüentemente despercebidas no contexto médico e pacientes clínicos deprimidos recebem tratamento inadequado para sua doença afetiva. 


\section{Referências}

Andersen G, Vestergaard K, Lauritzen L. Effective treatment of poststroke depression with the selective serotonin reuptake inhibitor citalopram. Stroke, 25:1099-104, 1994.

Andersen J, Aabro E, Gulmann N, Hjelmsted A, Pedersen HE. Antidepressive treatment in Parkinson's disease. A controlled trial of the effect of nortriptyline in patients with Parkinson's disease treated with L-DOPA. Acta Neurol Scand, 62: 210-9, 1980.

Appolinario JC, Bueno JR, Coutinho W. Psychotropic drugs in the treatment of obesity: what promise? CNS Drugs, 18: 629-51, 2004

Arinzon ZH, Lehman YA, Fidelman ZG, Krasnyansky, II. Delayed recurrent SIADH associated with SSRIs. Ann Pharmacother 36: 1175-7, 2002

Blazer DG, Hughes D, George LK, Swartz M, Boyer R. Generalized anxiety disorder. In: Robins LN, Regier DA. Psychiatry disorders in America: the epidemiologic catchment area study. Free Press, p. 180-203, New York, 1993.

Block S, Kissane. Psychotherapies in psycho-oncology: an exciting new challenge. British Journal of Psychiatry, 177: 112-6, 2000.

Botega NJ, Bio MR, Zomignani MA, Garcia C, Jr., Pereira WA. Mood disorders among inpatients in ambulatory and validation of the anxiety and depression scale HAD. Rev Saúde Pública, 29: 355-63, 1995.

Brasil MAA. Medicamentos. In: Fráguas Júnior R, Figueiredo JAB, editores. Depressões em medicina interna e outras condições. Depressões secundárias. Atheneu, p. 333-45, São Paulo, 2001.

Brasil MAA. Pacientes com queixas difusas: um estudo nosológico de pacientes com queixas múltiplas e vagas. Tese de doutorado. Instituto de Psiquiatria da UFRJ, 1995.

Brasil MAA, Furlanetto LM. A atual nosologia psiquiátrica e sua adequação aos hospitais gerais. Cadernos do IPUB, Saúde mental no hospital geral, p. 59-70, 1997.

Carson AJ, MacHale S, Allen K, et al. Depression after stroke and Iesion location: a systematic review. Lancet, 356: 122-6, 2000.

Chochinov HM, Wilson KG, Enns M, Lander S. Prevalence of depression in the terminally ill: effects of diagnostic criteria and symptom threshold judgments. Am J Psychiatry, 151: 537-40, 1994

Cigognini MA, Furlanetto LM. Treatment of depressive patients in a general hospital. Rev Bras Psiquiatr, 28 (no prelo), 2006.

Clarke DM, Kissane DW. Demoralization: its phenomenology and importance. Aust NZJ Psychiatry, 36: 733-42, 2002.

Dalton SO, Johansen C, Mellemkjaer L, Norgard B, Sorensen HT, Olsen JH. Use of selective serotonin reuptake inhibitors and risk of upper gastrointestinal tract bleeding: a population-based cohort study. Arch Intern Med, 163: 59-64, 2003.

de Groot M, Anderson R, Freedland KE, Clouse RE, Lustman PJ. Association of depression and diabetes complications: a metaanalysis. Psychosom Med, 63: 619-30, 2001.

de Jonge P, Kempen GI, Sanderman R, et al. Depressive symptoms in elderly patients after a somatic illness event: prevalence, persistence and risk factors. Psychosomatics, 47: 33-42, 2006a.

de Jonge P, Spijkerman TA, van den Brink RH, Ormel J. Depression after myocardial infarction is a risk factor for declining health related quality of life and increased disability and cardiac complaints at 12 months. Heart, 92: 32-9, 2006b.
Degner D, Grohmann R, Kropp S, et al. Severe adverse drug reactions of antidepressants: results of the German multicenter drug surveillance program AMSP. Pharmacopsychiatry, 37 Suppl 1: S39-45, 2004.

Ehlert U, Gaab J, Heinrichs M. Psychoneuroendocrinological contributions to the etiology of depression, posttraumatic stress disorder and stress-related bodily disorders: the role of the hypothalamus-pituitary-adrenal axis. Biol Psychol, 57: 141-52, 2001

Eisch AJ, Nestler EJ. To be or not to be: adult neurogenesis and psychiatry. Clin Neuroscience Res, 2: 93-108, 2002.

Endicott J. Measurement of depression in patients with cancer. Cancer, 53: 2243-9, 1984.

Evans DL, Charney DS, Lewis L, et al. Mood disorders in the medically ill: scientific review and recommendations. Biol Psychiatry, 58 : 175-89, 2005

Everson SA, Kaplan GA, Goldberg DE, Salonen JT. Hypertension incidence is predicted by high levels of hopelessness in Finnish men. Hypertension, 35: 561-7, 2000

Farrell KR, Ganzini L. Misdiagnosing delirium as depression in medically ill elderly patients. Arch Intern Med, 155: 2459-64, 1995.

Ferrando SJ, Rabkin JG, de Moore GM, Rabkin R. Antidepressant treatment of depression in HIV-seropositive women. J Clin Psychiatry, 60: 741-6, 1999.

Figueiredo JM. Depression and demoralization: phenomenologic differences and research perspectives. Compr Psychiatry, 34: 308-11, 1993

Figueiredo JM. Some issues in research on the epidemiology of demoralization. Compr Psychiatry, 24: 154-7, 1983.

Figueiredo, JM, Frank JD. Subjetive incompetence, the clinical hallmark of demoralization. Compr Psychiatry, 23: 353-63, 1982.

Fishbain D. Evidence-based data on pain relief with antidepressants. Ann Med, 32: 305-16, 2000.

Fleck MAP, Soares HH. Estudo preliminar da prevalência de alcoolismo em pacientes internados no pronto-socorro de Porto Alegre. Rev Psiquiatr RS, 12: 84-7, 1990.

Fornari LM, Furlanetto LM. Freqüência de sintomas depressivos em pacientes com história de infarto agudo do miocárdio. J Bras Psiquiatr, 51: 385-90, 2002.

Fortes S. Transtornos mentais na atenção primária: suas formas de apresentação, perfil nosológico e fatores associados em unidades do Programa de Saúde da Família do município de Petrópolis, Rio de Janeiro, Brasil. 2004. Tese (doutorado). Universidade do Estado do Rio de Janeiro, Rio de Janeiro.

Fráguas Jr. R, Gonsalves Henriques Jr. S, De Lucia MS, et al. The detection of depression in medical setting: a study with PRIMEMD. J Affect Disord, 91: 11-7, 2006.

Frasure-Smith N, Lesperance F, Talajic M. Depression following myocardial infarction. Impact on 6-month survival. JAMA, 270: 1819-25, 1993

Furlanetto LM. Diagnosticando depressão em pacientes internados em enfermarias de clínica médica. J Bras Psiq, 45: 363-70, 1996.

Furlanetto LM. Aspectos metodológicos da pesquisa em interconsulta psiquiátrica. J Bras Psiq, 46: 371-6, 1997a.

Furlanetto LM. Depressão mascarada existe? Rev Bras Med Psicossom, 1, 1997b. 
Furlanetto LM. Diagnóstico. In: Fráguas Júnior R, Figueiredo JAB, editores. Depressões em medicina interna e em outras condições médicas. Depressões secundárias. Atheneu, p. 11-20, Rio de Janeiro, 2000.

Furlanetto LM, Bueno JR, Silva RV. Características e evolução de pacientes com transtornos depressivos durante a internação em enfermarias de clínica médica. J Bras Psiq, 47: 609-17, 1998.

Furlanetto LM, Mendlowicz MV, Romildo Bueno J. The validity of the Beck Depression Inventory - Short Form as a screening and diagnostic instrument for moderate and severe depression in medical inpatients. J Affect Disord, 86: 87-91, 2005.

Furlanetto LM, von Ammon Cavanaugh S, Bueno JR, Creech SD, Powell LH. Association between depressive symptoms and mortality in medical inpatients. Psychosomatics, 41: 426-32, 2000.

Gainotti G, Antonucci G, Marra C, Paolucci S. Relation between depression after stroke, antidepressant therapy and functional recovery. J Neurol Neurosurg Psychiatry, 71: 258-61, 2001.

Gauer GJC, Furlanetto LM, Juruema MF, Vieira RM. Transtornos do humor. In: Neto AC, Gauer GJC, Furtado NR, editores. Psiquiatria para Estudantes de Medicina. Edipucrs, p. 386-406, Porto Alegre, 2003.

Glassman AH, O'Connor CM, Califf RM, et al. Sertraline treatment of major depression in patients with acute $\mathrm{MI}$ or unstable angina. JAMA, 288: 701-9, 2002.

Goldberg DP, Huxley P. Common mental disorders: a bio-social model. Routledge, London,1992.

Golden SH, Williams JE, Ford DE, et al. Depressive symptoms and the risk of type 2 diabetes: the atherosclerosis risk in communities study. Diabetes Care, 27: 429-35, 2004.

Gonzalez-Torrecillas JL, Mendlewicz J, Lobo A. Effects of early treatment of poststroke depression on neuropsychological rehabilitation. Int Psychogeriatr, 7: 547-60, 1995.

Green RC, Cupples LA, Kurz A, et al. Depression as a risk factor for Alzheimer disease: the MIRAGE Study. Arch Neurol, 60: 753-9, 2003.

Griffith JL, Gaby L. Brief psychotherapy at the bedside: countering demoralization from medical illness. Psychosomatics, 46(2): 109-16, 2005.

Hesdorffer DC, Hauser WA, Annegers JF, Cascino G. Major depression is a risk factor for seizures in older adults. Ann Neurol, 47: 2469, 2000.

Holland JC, Romano SJ, Heiligenstein JH, Tepner RG, Wilson MG. A controlled trial of fluoxetine and desipramine in depressed women with advanced cancer. Psychooncology, 7: 291-300, 1998.

House A, Knapp P, Bamford J, Vail A. Mortality at 12 and 24 months after stroke may be associated with depressive symptoms at 1 month. Stroke, 32: 696-701, 2001.

Jamison KR. The many stigmas of mental illness. Lancet, 367: 5334, 2006.

Kessler RC, Demler O, Frank RG, et al. Prevalence and treatment of mental disorders, 1990 to 2003. N Engl J Med, 352: 2515-23, 2005.

Koenig HG, George LK, Meador KG. Use of antidepressants by nonpsychiatrists in the treatment of medically ill hospitalized depressed elderly patients. Am J Psychiatry, 154: 1369-75, 1997a.
Koenig HG, George LK, Peterson BL, Pieper CF. Depression in medically ill hospitalized older adults: prevalence, characteristics, and course of symptoms according to six diagnostic schemes. Am J Psychiatry, 154: 1376-83, 1997b.

Kop WJ, Gottdiener JS. The role of immune system parameters in the relationship between depression and coronary artery disease. Psychosom Med, 67 Suppl 1: S37-41, 2005.

Kop WJ, Gottdiener JS, Tangen CM, et al. Inflammation and coagulation factors in persons $>65$ years of age with symptoms of depression but without evidence of myocardial ischemia. Am J Cardiol, 89: 419-24, 2002.

Kumar A, Thomas A, Lavretsky $\mathrm{H}$, et al. Frontal white matter biochemical abnormalities in late-life major depression detected with proton magnetic resonance spectroscopy. Am J Psychiatry, 159: 630-6, 2002.

Kunik M, Braun U, Stanley M, et al. One session cognitive behavioural therapy for elderly patients with chronic obstructive pulmonary disease. Psychological Medicine, 31: 717-23, 2001.

Kuo HK, Yen CJ, Chang CH, Kuo CK, Chen JH, Sorond F. Relation of C-reactive protein to stroke, cognitive disorders, and depression in the general population: systematic review and meta-analysis. Lancet Neurol, 4: 371-80, 2005.

Laghrissi-Thode F, Wagner WR, Pollock BG, Johnson PC, Finkel MS. Elevated platelet factor 4 and beta-thromboglobulin plasma levels in depressed patients with ischemic heart disease. Biol Psychiatry, 42: 290-5, 1997.

Larson SL, Owens PL, Ford D, Eaton W. Depressive disorder, dysthymia, and risk of stroke: thirteen-year follow-up from the Baltimore epidemiologic catchment area study. Stroke, 32: 1979-83, 2001.

Leibing,E, Pfingsten M, Bartmann U, et al. Cognitive-behavioural treatment in unslected rheumatoid arthritis outpatients. Clin J Pain, 15: 58-66, 1999.

Leserman J, Petitto JM, Gu H, et al. Progression to AIDS, a clinical AIDS condition and mortality: psychosocial and physiological predictors. Psychol Med, 32: 1059-73, 2002.

Lesperance F, Frasure-Smith N, Juneau M, Theroux P. Depression and 1-year prognosis in unstable angina. Arch Intern Med, 160: 1354-60, 2000.

Lesperance F, Frasure-Smith N, Talajic M. Major depression before and after myocardial infarction: its nature and consequences. Psychosom Med, 58: 99-110, 1996.

Lesperance F, Frasure-Smith N, Talajic M, Bourassa MG. Five-year risk of cardiac mortality in relation to initial severity and one-year changes in depression symptoms after myocardial infarction. Circulation, 105: 1049-53, 2002.

Lin EH, Katon W, Von Korff M, et al. Relationship of depression and diabetes self-care, medication adherence, and preventive care. Diabetes Care, 27: 2154-60, 2004.

Lipsey JR, Robinson RG, Pearlson GD, Rao K, Price TR. Nortriptyline treatment of post-stroke depression: a double-blind study. Lancet, 1: 297-300, 1984.

Lustman PJ, Clouse RE. Depression in diabetic patients: the relationship between mood and glycemic control. J Diabetes Complications, 19: 113-22, 2005.

Lustman PJ, Freedland KE, Griffith LS, Clouse RE. Fluoxetine for depression in diabetes: a randomized double-blind placebocontrolled trial. Diabetes Care, 23: 618-23, 2000. 
Lustman PJ, Griffith LS, Clouse RE, et al. Effects of nortriptyline on depression and glycemic control in diabetes: results of a double-blind, placebo-controlled trial. Psychosom Med, 59: 241-50, 1997.

Lyketsos CG, DelCampo L, Steinberg M, et al. Treating depression in Alzheimer disease: efficacy and safety of sertraline therapy and the benefits of depression reduction: the DIADS. Arch Gen Psychiatry, 60: 737-46, 2003.

Mangelli L, Fava GA, Grandi S, Grassi L, Ottolini F, Porcelli P, et al. Assessing demoralization and depression in the setting of medical disease. J Clin Psychiatry, 66(3): 391-4, 2005.

Manji H. Depression, III: treatments. Am J Psychiatry, 160: 24, 2003.

Miller GE, Stetler CA, Carney RM, Freedland KE, Banks WA. Clinical depression and inflammatory risk markers for coronary heart disease. Am J Cardiol, 90: 1279-83, 2002.

Montano CB. Recognition and treatment of depression in a primary care setting. J Clin Psychiatry, 55 Suppl: 18-34; discussion 35-7, 1994.

Montejo AL, Llorca G, Izquierdo JA, Rico-Villademoros F. Incidence of sexual dysfunction associated with antidepressant agents: a prospective multicenter study of 1022 outpatients. Spanish Working Group for the Study of Psychotropic-Related Sexual Dysfunction. J Clin Psychiatry, 62 Suppl 3: 10-21, 2001

Mossner R, Henneberg A, Schmitt A, et al. Allelic variation of serotonin transporter expression is associated with depression in Parkinson's disease. Mol Psychiatry, 6: 350-2, 2001.

Musselman DL, Betan E, Larsen H, Phillips LS. Relationship of depression to diabetes types 1 and 2: epidemiology, biology, and treatment. Biol Psychiatry, 54: 317-29, 2003.

Musselman DL, Lawson DH, Gumnick JF, et al. Paroxetine for the prevention of depression induced by high-dose interferon alfa. N Engl J Med, 344: 961-6, 2001

Nobuhara K, Okugawa G, Sugimoto T, et al. Frontal white matter anisotropy and symptom severity of late-life depression: a magnetic resonance diffusion tensor imaging study. J Neurol Neurosurg Psychiatry, 77: 120-2, 2006.

Petracca GM, Chemerinski E, Starkstein SE. A double-blind placebo-controlled study of fluoxetine in depressed patients with Alzheimer's disease. Int Psychogeriatr, 13: 233-40, 2001.

Pezzella G, Moslinger-Gehmayr R, Contu A. Treatment of depression in patients with breast cancer: a comparison between paroxetine and amitriptyline. Breast Cancer Res Treat, 70: 1-10, 2001.

Polsky D, Doshi JA, Marcus S, et al. Long-term risk for depressive symptoms after a medical diagnosis. Arch Intern Med, 165: 1260-6, 2005.

Pratt LA, Ford DE, Crum RM, Armenian HK, Gallo JJ, Eaton WW. Depression, psychotropic medication, and risk of myocardial infarction. Prospective data from the Baltimore ECA follow-up. Circulation, 94: 3123-9, 1996.

Rabkin JG, Wagner GJ, Rabkin R. Fluoxetine treatment for depression in patients with HIV and AIDS: a randomized, placebo-controlled trial. Am J Psychiatry, 156: 101-7, 1999.

Rafanelli C, Roncuzzi R, Milaneschi Y, Tomba E, Colistro MC, Pancald LG, et al. Stressful life events, depression and demoralization as risk factors for acute coronary heart disease. Psychother Psychosom, 74(3): 179-84, 2005.

Roberts RE, Deleger S, Strawbridge WJ, Kaplan GA. Prospective association between obesity and depression: evidence from the Alameda County Study. Int J Obes Relat Metab Disord, 27: 514-21, 2003.
Robinson RC, Rabins PV. Depression and coexisting disease. IgakuShoin, New York, 1989.

Robinson RG. Poststroke depression: prevalence, diagnosis, treatment, and disease progression. Biol Psychiatry, 54: 37687, 2003

Robinson RG, Schultz SK, Castillo C, et al. Nortriptyline versus fluoxetine in the treatment of depression and in short-term recovery after stroke: a placebo-controlled, double-blind study. Am J Psychiatry, 157: 351-9, 2000

Rumsfeld JS, Jones PG, Whooley MA, et al. Depression predicts mortality and hospitalization in patients with myocardial infarction complicated by heart failure. Am Heart J, 150: 961-7, 2005.

Rodin G, Craven J, Littlefield C. Depression in the medically ill. Brunner\&Mazel, New York, 1991

Rundell JR, Wise MG. The American Psychiatric Press textbook of consultation-liaison psychiatry. American Psychiatric Press, Washington, 2005.

Schiepers OJ, Wichers MC, Maes M. Cytokines and major depression. Prog Neuropsychopharmacol Biol Psychiatry, 29: 201-17, 2005.

Schildkraut JJ, Klein FD. The classification and treatment of depressive states. In: Shader RI. Manual of Psychiatric Therapeutics. Little\&Brown, Boston, 1975.

Shader RI. Demoralization revisited. J Clin Psychopharmacology, 25(4): 291-2, 2005

Sherwood A, Hinderliter AL, Watkins LL, Waugh RA, Blumenthal JA. Impaired endothelial function in coronary heart disease patients with depressive symptomatology. J Am Coll Cardiol, 46: 656-9, 2005.

Simen BB, Duman CH, Simen AA, Duman RS. TNF-alpha signaling in depression and anxiety: behavioral consequences of individual receptor targeting. Biol Psychiatry, 2006.

Simon GE, Von Korff M. Medical co-morbidity and validity of DSM-IV depression criteria. Psychol Med, 36: 27-36, 2006.

Spiegel D, Giese-Davis J. Depression and cancer: mechanisms and disease progression. Biol Psychiatry, 54: 269-82, 2003.

Strain JJ. Psychiatric diagnostic dilemmas in the medical setting. Aust N Z J Psychiatry, 39: 764-71, 2005.

Sullivan MD, LaCroix AZ, Baum C, Grothaus LC, Katon WJ. Functional status in coronary artery disease: a one-year prospective study of the role of anxiety and depression. Am J Med, 103: 348-56, 1997.

Taylor CB, Youngblood ME, Catellier D, et al. Effects of antidepressant medication on morbidity and mortality in depressed patients after myocardial infarction. Arch Gen Psychiatry, 62: 792-8, 2005.

Unutzer J, Patrick DL, Simon G, et al. Depressive symptoms and the cost of health services in HMO patients aged 65 years and older. A 4-year prospective study. JAMA, 277: 1618-23, 1997.

Villano LA, Nanhay ALG, Moraes LR, Costa e Silva JA. Results from the Rio de Janeiro Centre in Mental Illness in General Health Care: an International Study. In: Ustün TB, Sartorius N, editors. John Wiley \& Sons Ltd, 1995.

von Ammon Cavanaugh S. Depression in the medically ill. Critical issues in diagnostic assessment. Psychosomatics, 36: 48-59, 1995.

von Ammon Cavanaugh S, Furlanetto LM, Creech SD, Powell LH. Medical illness, past depression and present depression: a predictive triad for in-hospital mortality. Am J Psychiatry, 158: 43-8, 2001 
Wang PS, Bohn RL, Knight E, Glynn RJ, Mogun H, Avorn J. Noncompliance with antihypertensive medications: the impact of depressive symptoms and psychosocial factors. J Gen Intern Med, 17: 504-11, 2002.

Wells K, Sherbourne C, Duan N, et al. Quality improvement for depression in primary care: do patients with subthreshold depression benefit in the long run? Am J Psychiatry, 162: 114957, 2005.

White CA. Cognitive behavior therapy for chronic medical problems: a guide to assessment and treatment in practice. John Wiley, West Sussex, 2001.

Wiart L, Petit H, Joseph PA, Mazaux JM, Barat M. Fluoxetine in early poststroke depression: a double-blind placebo-controlled study. Stroke, 31: 1829-32, 2000.

Wichers MC, Kenis G, Leue C, Koek G, Robaeys G, Maes M. Baseline immune activation as a risk factor for the onset of depression during interferon-alpha treatment. Biol Psychiatry (no prelo), 2006.

Yasui-Furukori N, Kaneko S. Digitalis intoxication induced by paroxetine co-administration. Lancet, 367: 788, 2006.

Zhou S, Chan E, Pan SQ, Huang M, Lee EJ. Pharmacokinetic interactions of drugs with St. John's wort. J Psychopharmacol, 18: 262-76, 2004.

Ziegelstein RC, Fauerbach JA, Stevens SS, Romanelli J, Richter DP, Bush DE. Patients with depression are less likely to follow recommendations to reduce cardiac risk during recovery from a myocardial infarction. Arch Intern Med, 160: 1818-23, 2000.

Ziegelstein RC. Depression in patients recovering from a myocardial infarction. JAMA, 286(13): 1621-7, 2001.

Zubenko GS, Zubenko WN, McPherson S, et al. A collaborative study of the emergence and clinical features of the major depressive syndrome of Alzheimer's disease. Am J Psychiatry, 160: 857-66, 2003. 